Objective To estimate the incidence and proportion of noncontact to total ACL injuries by sex, age, sport, participation level, and exposure type.

Design Systematic review, meta-analysis and meta-regression Setting Team ball-sports (soccer, rugby, basketball, volleyball, American and Australian football, floorball, lacrosse, hockey, baseball, softball)

Participants Athletes participating in team ball-sports regardless of sex, age, and participation level

Interventions Six electronic databases were searched from inception to July 2020. Cohort studies of team ball-sports reporting number of knee injuries as a function of exposure and injury mechanism were included.

Main Outcome Measurements Proportion of non-contact to total ACL injuries and non-contact ACL injury incidence by unit of exposure, sex, age group, sport, participation level, and exposure type.

Results Forty-three studies covering 12 sports were included. The overall proportion of non-contact to total ACL injuries was $54 \%\left(95 \%\right.$ CI $47-60 \%, I^{2}=90 \%$; females: $62 \%, 95 \%$ CI 54-70\%, $I^{2}=83 \%$; males: 47\%, 95\% CI 39-54\%, $\left.I^{2}=86 \%\right)$. Injury incidence was higher in females $(0.20$ per 1000 player-hours, 95\% CI $0.12-0.33, I^{2}=92 \%$; 0.07 per 1000 player-exposures, 95\% CI $\left.0.04-0.14, I^{2}=98 \%\right)$ than males (0.08 per 1000 player-hours, 95\% CI $0.04-0.14, I^{2}=94 \%$; 0.05 per 1000 player-exposures, 95\% CI 0.03-0.08, $\left.I^{2}=97 \%\right)$. Injury incidence during competition was much higher $(0.53$ per 1000 player-hours, 95\% CI $0.35-0.81$, $I^{2}=80 \%$; 0.35 per 1000 player-exposures, 95\% CI $0.17-$ $\left.0.71, I^{2}=96 \%\right)$ than training $(0.03$ per 1000 player-hours (95\% CI $0.02-0.05, I^{2}=60 \%$; 0.02 per 1000 player-exposures, 95\% CI $0.01-0.05, I^{2}=86 \%$ ). Heterogeneity between studies was generally high.

Conclusions Non-contact ACL injuries represented over half of all ACL injuries sustained. The proportion of non-contact to total ACL injuries and injury incidence were higher in females than males. Injuries mostly occurred in competition settings. More research is required to fully understand the influence of sport, age group, and participation level on injury proportion and incidence. difficult.

\section{LONGITUDINAL CHANGES IN CUTTING KINEMATICS IN YOUNG FEMALE ATHLETES ACROSS PUBERTY}

${ }^{1}$ Lionel Chia, ${ }^{2}$ Timothy Hewett, ${ }^{3}$ Kevin Ford, ${ }^{4,5}$ Gregory Myer, ${ }^{1}$ Marnee McKay, ${ }^{1}$ Justin Sullivan, ${ }^{1,6}$ Pappas Evangelos. 'Sydney School of Health Sciences, The University of Sydney, NSW, Australia; ${ }^{2}$ Hewett Global Consulting, MN, USA; ${ }^{3}$ Department of Physical Therapy, High Point University, NC, USA; ${ }^{4}$ Emory Sport Performance and Research Center, GA, USA; ${ }^{5}$ Emory University School of Medicine, GA, USA; ${ }^{6}$ University of Wollongong, NSW, Australia

\subsection{6/bjsports-2021-IOC.365}

Background Young female athletes involved in high-speed cutting and change-of-direction sports are particularly susceptible to anterior cruciate ligament (ACL) injuries. Little is known if maturational changes in cutting technique contribute to the increased injury risk.

Objective To examine longitudinal changes in cutting kinematics in female athletes as they matured through puberty.

Design Longitudinal cohort study

Setting Biomechanics laboratory
Patients (or Participants) One-hundred and seventy-two healthy high-school athletes were tested while in at least two different pubertal stages (pre-, mid-, post-pubertal).

Interventions (or Assessment of Risk Factors) Trunk, hip, and knee sagittal, frontal and transverse plane range-of-motion, peak angles, and angles at initial-contact during an unanticipated $45^{\circ}$ cut measured with a laboratory-based optoelectronic motion capture system. Pubertal stages were defined by Tanner Stages.

Main Outcome Measurements Changes in trunk, hip, and knee kinematics as girls mature across puberty (pre-to-midpubertal, pre-to-postpubertal, mid-to-postpubertal).

Results As girls matured through puberty, they displayed a decrease in sagittal-plane hip $\left(1.6-4.4^{\circ}, \mathrm{p} \leq 0.01\right)$ and knee range-of-motion (ROM) $\left(2.7-2.9^{\circ}, \mathrm{p} \leq 0.01\right)$, and decreased peak hip $\left(2.9-3.2^{\circ}, \mathrm{p} \leq 0.02\right)$ and knee flexion angles $\left(2.5-2.9^{\circ}\right.$, $\mathrm{p} \leq 0.01$ ), which is indicative of greater quadriceps dominance. Peak knee abduction angles also increased as girls progressed through puberty $\left(0.9-1.4^{\circ}, \mathrm{p} \leq 0.006\right)$, suggesting greater ligament dominance. In terms of trunk dominance, there were mixed findings with a decrease in trunk frontal- $\left(2.5-5.7^{\circ}\right.$, $\mathrm{p}<0.01)$ and sagittal-plane ROM $\left(1.4-2.0^{\circ}, \mathrm{p} \leq 0.05\right)$, but an increase in trunk transverse-plane ROM $\left(2.8-3.6^{\circ}, \mathrm{p} \leq 0.01\right)$ observed as girls mature. Other significant changes in cutting technique were decreased peak trunk flexion $\left(3.8-7.8^{\circ}\right.$, $\mathrm{p} \leq 0.01)$, and decreased hip flexion $\left(2.9-3.3^{\circ}, \mathrm{p} \leq 0.01\right)$ and knee flexion angles $\left(2.0-3.0^{\circ}, \mathrm{p} \leq 0.02\right)$ at initial contact, suggesting a more upright and stiffer cutting posture.

Conclusions As girls mature through puberty, they display a change in cutting strategy characterized by greater quadriceps and ligament dominance. Ameliorating these kinematic changes throughout maturation may further reduce ACL injuries in this high-risk group.

\section{BONE BRUISE PATTERN AND MECHANISM OF ANTERIOR CRUCIATE LIGAMENT INJURY IN PROFESSIONAL FOOTBALL PLAYERS: CORRELATION BETWEEN MRI AND VIDEO ANALYSIS}

${ }^{1}$ Pieter D'Hooghe, ${ }^{2}$ Alberto Grassi, ${ }^{3}$ Francesco Della Villa, ${ }^{1}$ Khalid Alkhelaifi, ${ }^{1}$ Emmanouil Papakostas, ${ }^{2}$ Filippo Tosarelli, ${ }^{2}$ Stefano Zaffagnini. ${ }^{1}$ Aspetar Hospital, Doha, Qatar; ${ }^{2}$ IRCCS Istituto Ortopedico Rizzoli, Bologna, Italy; ${ }^{3}$ Isokinetic Group, Bologna, Italy

\subsection{6/bjsports-2021-IOC.366}

Background The presence of bone bruises (BB), especially in the lateral compartment of the knee, is a common finding in the context of anterior cruciate ligament (ACL) injury. However, different patterns have been described and a precise correlation with mechanism of ACL injury has never been determined.

Objective To correlate the MRI features of ACL injuries with the exact injury mechanism obtained from video-analysis. Such investigation, which has never been performed up to now, provides relevant insights in the understanding ACL injury mechanism and in the genesis of intra-articular lesions such as bone bruises.

Design Fifteen professional football players sustaining an ACL injury (while playing during an official match of First League Championship) were included in the study. The video of injury was obtained from the Television broadcast. Knee Magnetic Resonance Imaging (MRI) was obtained within 7 days 